\title{
PERIODIC SOLUTIONS OF FINITE DIFFERENCE EQUATIONS*
}

\author{
$\mathrm{Br}$ \\ M. BRAUN AND J. HERSHENOV \\ Queens College (CUNY), Flushing, N. Y.
}

Introduction. It is well known that the qualitative properties of solutions of finite difference approximations of an ordinary differential equation may differ greatly from the qualitative properties of the solutions of the differential equation. A classic example is the differential equation

$$
\ddot{x}+x=0 .
$$

If we set $y_{1}=x$ and $y_{2}=\dot{x}$, then the Euler approximation of (1) is

$$
y_{k+1}=A y_{k}=\left(\begin{array}{rr}
1 & h \\
-h & 1
\end{array}\right) y_{k} .
$$

The eigenvalues of the matrix $A$ are $1 \pm h i$. Hence all the iterates $y_{1}, y_{2}, \cdots$, of (2) spiral away from the origin. On the other hand, every non-trivial solution of (1) is periodic with period $2 \pi$.

The essential difficulty with the differential equation (1) is that the equilibrium solution $x=\dot{x}=0$ is a center. Equivalently, the periodic solutions of (1) are not orbitally asymptotically stable, and thus they may be destroyed under an arbitrary small perturbation. The situation is very different, however, when $x=\phi(t)\left(x=\left(x_{1}, \cdots, x_{n}\right)\right)$ is an orbitally asymptotically stable (unstable) solution of the differential equation

$$
\dot{x}=f(x) \text {. }
$$

Let

$$
x_{k+1}=F\left(x_{k}, h\right)
$$

be a finite difference approximation of (3). While we cannot expect the difference equation (4) to possess a closed invariant curve of periodic points, we would expect that the difference equation (4) has a closed invariant curve $\Gamma$ near $x=\phi(t)$, and that the solutions $x_{k}$ of (4) spiral into $\Gamma$ as $k \rightarrow \infty(-\infty)$. This was verified recently for the special case of the Van der Pol equation

$$
\ddot{x}+\epsilon\left(x^{2}-1\right) \dot{x}+x=0 .
$$

It is well known (see Hale [4]) that this equation has a periodic solution $x=\phi(t)$ with $\phi^{2}+\dot{\phi}^{2} \simeq 2$, for $\epsilon$ sufficiently small, and positive. Using theorems of Halanay [2], [3] on the existence of periodic solutions of difference equations, and a suitable integral

* Received March 19, 1976. This research was partially supported by NSF Grant MPS 74-07502. 
manifold method, Hurt [5] was able to show that the central difference analogue

$$
\frac{1}{h^{2}}\left[x\left(t_{k+1}\right)-2 x\left(t_{k}\right)+x\left(t_{k-1}\right)\right]+\epsilon\left(x^{2}\left(t_{k}\right)-1\right) \frac{\left(x\left(t_{k+1}\right)-x\left(t_{k-1}\right)\right)}{2 h}+x\left(t_{k}\right)=0
$$

to (5) has a closed invariant curve near $x=\phi(t)$ for $\epsilon>0$ and $h$ sufficiently small.

In this paper we generalize Hurt's result to the case of any orbitally asymptotically stable (unstable) periodic solution of any autonomous differential equation. In particular, we prove the following theorem:

Theorem 1: Let $x=\phi(t)$ be a periodic solution of (3), with characteristic multipliers $\mu_{1}, \cdots, \mu_{n-1}, \mu_{n}=1$ and let

$$
x_{k+1}=F\left(x_{k}, h\right)
$$

be a finite difference approximation of (3) which is at least as accurate as Euler's method. Assume that either $\left|\mu_{j}\right|<1, j=1, \cdots, n-1$ or $\left|\mu_{i}\right|>1, j=1, \cdots, n-1$. Then the difference equation (6) has a closed invariant curve $\Gamma$ near $x=\phi(t)$, for $h$ small. Furthermore, the iterates $x_{k}$ of $x_{0}$ sprial into $\Gamma$ as $k \rightarrow \infty$, or $-\infty$, for $x_{0}$ sufficiently close to $\Gamma$.

As a corollary to this theorem, we show that the second characteristic multiplier of the periodic solution $x=\phi(t)$ of the Van der Pol equation (5) has modulus less than one. Hence, any finite difference approximation of (5) which is at least as accurate as Euler's method has a closed invariant curve $\Gamma$ near $x=\phi(t)$, for $h$ small, and all the iterates $x_{k}$ of $x_{0}$ spiral into $\Gamma$, for $x_{0}$ sufficiently close to $\Gamma$. This result answers all of the questions raised in [5].

The mapping $M^{N}$. For simplicity of writing and notational convenience, we will prove Theorem 1 for the case $n=2$, and then show how the proof generalizes to arbitrary $n$. Thus we assume that the differential equation

$$
\dot{x}=f(x, y), \quad \dot{y}=g(x, y)
$$

has a periodic solution $x=\phi(t), y=\psi(t)$, with characteristic multipliers $\mu_{1}=1$ and $\mu_{2}$, with $\left|\mu_{2}\right| \neq 1$. The functions $f$ and $g$ have three continuous derivatives with respect to $x$ and $y$. Then, any finite difference approximation of (7) which is at least as accurate as Euler's method can be written in the form

$$
\begin{aligned}
& x_{k+1}=x_{k}+h f\left(x_{k}, y_{k}\right)+h^{2} \hat{f}\left(x_{k}, y_{k}, h\right) \\
& y_{k+1}=y_{k}+h g\left(x_{k}, y_{k}\right)+h^{2} \hat{g}\left(x_{k}, y_{k}, h\right)
\end{aligned}
$$

The key step in proving Theorem 1 is to introduce "normal coordinates" $r, \theta$ near the periodic solution $x=\phi(t), y=\psi(t)$ of (7). Following Hale [4], we transform this periodic solution to the circle $r=0$ by a suitable transformation $r=u(x, y), \theta=v(x, y)$. This can be done in such a way that

$$
\begin{aligned}
& \dot{r}=u_{x} f+u_{\nu} g=a(\theta) r+f_{1}(r, \theta) \\
& \dot{\theta}=v_{x} f+v_{\nu} g=1+f_{2}(r, \theta)
\end{aligned}
$$

where

$$
f_{2}(0, \theta)=f_{1}(0, \theta)=\left(\partial f_{1} / \partial r\right)(0, \theta)=0
$$


and $\exp \left(\int_{0}{ }^{2 r} a(\theta) d \theta\right)$ is the characteristic multiplier $\mu_{2}$. Applying this transformation to the difference equation (8) gives

$$
\begin{aligned}
r_{k+1} & =u\left(x_{k+1}, y_{k+1}\right) \\
& =u\left(x_{k}+h f\left(x_{k}, y_{k}\right)+h^{2} \hat{f}, y_{k}+h g\left(x_{k}, y_{k}\right)+h^{2} \hat{g}\right) \\
& =u\left(x_{k}, y_{k}\right)+h\left(u_{x} f+u_{y} g\right)+h^{2} \hat{F}_{1} \\
& =r_{k}+h a\left(\theta_{k}\right) r_{k}+h f_{1}\left(r_{k}, \theta_{k}\right)+h^{2} F_{1}\left(r_{k}, \theta_{k}, h\right) .
\end{aligned}
$$

Similarly,

$$
\begin{aligned}
\theta_{k+1} & =v\left(x_{k}+h f\left(x_{k}, y_{k}\right)+h^{2} \hat{f}, y_{k}+h g\left(x_{k}, y_{k}\right)+h^{2} \hat{g}\right) \\
& =\theta_{k}+h\left[1+f_{2}\left(r_{k}, \theta_{k}\right)\right]+h^{2} G_{1}\left(r_{k}, \theta_{k}, h\right) .
\end{aligned}
$$

Thus, we consider the mapping $M$

$$
M: \quad \begin{aligned}
r_{1} & =[1+h a(\theta)] r+h f_{1}(r, \theta)+h^{2} F_{1}(r, \theta, h) \\
\theta_{1} & =\theta+h f_{2}(r, \theta)+h^{2} G_{1}(r, \theta, h) .
\end{aligned}
$$

Together with $M$, we include the $N$ th iterate $M^{N}$ of $M$, where $N=[2 \pi / h]$, and we write $M^{N}$ in the form

$$
\begin{array}{ll}
r_{N} & =\lambda(\theta, r, h) r+F(r, \theta, h) \\
M^{N}: & \theta_{N}=\theta+2 \pi+G(r, \theta, h) .
\end{array}
$$

We will show that the mapping $M^{N}$ has a closed invariant curve $\Gamma$ near the circle $r=0$, and then we will show that $\Gamma$ is also invariant under $M$. It is very natural to consider the mapping $M^{N}$ and its iterates, because this is the section map that figures very prominently in the proof of the existence and stability of periodic solutions of differential equations.

Estimates of $\lambda, F$ and $G$. We first derive explicit representations for $\lambda, F$ and $G$. To this end, set

$$
a_{k}=1+h a\left(\theta_{k}\right), \quad b_{k}=h f_{1}\left(r_{k}, \theta_{k}\right)+h^{2} F_{1}\left(r_{k}, \theta_{k}, h\right) .
$$

Then (see Braun [1]),

$$
\begin{aligned}
r_{N}=a_{0} \cdots & a_{N-1} r+\sum_{i=0}^{N-1} a_{0} \cdots a_{N-1} \frac{b_{i}}{a_{0} \cdots a_{i}} \\
& =\prod_{i=0}^{N-1}\left[1+h a\left(\theta_{i}\right)\right] r+\left[\prod_{i=0}^{N-1}\left(1+h a\left(\theta_{i}\right)\right)\right] \sum_{i=0}^{N-1} \frac{h f_{1}\left(r_{i}, \theta_{i}\right)+h^{2} F_{1}\left(r_{i}, \theta_{i}, h\right)}{\prod_{\nu=0}^{i}\left[1+h a\left(\theta_{\nu}\right)\right]} .
\end{aligned}
$$

Hence,

$\lambda(\theta, r, h)=\prod_{i=0}^{N-1}\left[1+h a\left(\theta_{i}\right)\right], F(r, \theta, h)=\lambda \sum_{i=0}^{N-1} \frac{h f_{1}\left(r_{i}, \theta_{i}\right)+h^{2} F_{1}\left(r_{i}, \theta_{j}, h\right)}{\prod_{v=0}^{i}\left[1+h a\left(\theta_{v}\right)\right]}$.

Our first step is to estimate the iterates $r_{j}, j=1, \cdots, N-1$. To this end, choose a 
positive constant $c$ such that the functions $a(\theta), a^{\prime}(\theta), f_{1}(r, \theta) / r^{2}, f_{2}(r, \theta), F_{1}(r, \theta, h)$ and $G_{1}(r, \theta, h)$ are all less than or equal to $c$ in absolute value for $0 \leq \theta \leq 2 \pi,|r| \leq 1$, and $0 \leq h \leq 1$. (If necessary, we can rescale $r$ so that all these functions are defined for $|r| \leq R$, with $R>1$.) Then, we have the following lemma:

Lemma 1: Choose $r$ and $h$ sufficiently small that $|r|+h \leq \exp (-4 \pi c)$. Then,

$$
\left|r_{i}\right| \leq(1+2 h c)^{i}(|r|+h) \leq(\exp (4 \pi c))(|r|+h), \quad j=1, \cdots, N .
$$

Proof: From (11),

$$
\begin{aligned}
\left|r_{k+1}\right| & \leq(1+h c)\left|r_{k}\right|+h c\left|r_{k}\right|^{2}+c h^{2} \\
& \left.\leq(1+2 h c)\left|r_{k}\right|+c h^{2} \quad \text { (as long as }\left|r_{k}\right| \leq 1\right) .
\end{aligned}
$$

These inequalities imply that

$$
\begin{aligned}
\left|r_{i}\right| & \leq(1+2 h c)^{i}|r|+\frac{c h^{2}}{2 h c}\left[(1+2 h c)^{i}-1\right] \\
& \leq(1+2 h c)^{i}(|r|+h) \leq(\exp (2 h c j))(|r|+h) \\
& \leq(\exp (4 \pi c))(|r|+h)
\end{aligned}
$$

as long as $\left|r_{k}\right| \leq 1, k=1, \cdots, j$, and this in turn follows immediately if $|r|+h \leq$ $\exp (-4 \pi c)$.

Our next step is to show that $\lambda(r, \theta, h)=\prod_{i=0}^{N-1}\left(1+h a\left(\theta_{i}\right)\right)$ is approximately the second characteristic multiplier $\mu_{2}$ of the periodic solution $r=0$ of (9). In particular, if $\mu_{2}<1$, then $0<\lambda(r, \theta, h) \leq \rho<1$ uniformly for $0 \leq \theta \leq 2 \pi$, and $|r|$ and $h$ sufficiently small. This is the content of Lemmas 2 and 3.

Lemma 2:

$$
\operatorname{Limit}_{h \rightarrow 0} \frac{\prod_{i=0}^{N-1}\left(1+h a\left(\theta_{i}\right)\right)}{\exp \left(h \sum_{j=0}^{N-1} a\left(\theta_{i}\right)\right)}=1
$$

Proof: Observe that

$$
\exp \left(h a\left(\theta_{i}\right)\right)=1+h a\left(\theta_{i}\right)+e_{i}
$$

where

$$
\left|e_{i}\right| \leq \frac{h^{2} c^{2}}{2} \exp (h c)
$$

Hence,

$$
\prod_{i=0}^{N-1}\left(1+h a\left(\theta_{i}\right)\right)=\exp \left(h \sum_{j=0}^{N-1} a\left(\theta_{j}\right)\right) \prod_{j=0}^{N-1} 1-\exp \left(\left(-h a\left(\theta_{i}\right)\right) e_{i}\right)
$$

so that

$$
\prod_{i=0}^{N-1}\left(1-\frac{h^{2} c^{2}}{2} \exp (2 h c)\right) \leq \frac{\prod_{i=0}^{N-1}\left(1+h a\left(\theta_{i}\right)\right)}{\exp \left(h \sum_{i=0}^{N-1}\left(a \theta_{i}\right)\right)} \leq \prod_{i=0}^{N-1}\left(1+\frac{h^{2} c^{2}}{2} \exp (2 h c)\right)
$$


Lemma 2 now follows immediately from the trivial observation that

$$
\lim _{h \rightarrow 0}\left[1 \pm \frac{h^{2} c^{2}}{2} \exp (2 h c)\right]^{(2 \pi / h)}=1 .
$$

Lemma 3:

$$
\underset{|r|+h \rightarrow 0}{\operatorname{Limit}} \sum_{i=0}^{N-1} h\left[a(\theta+j h)-a\left(\theta_{i}\right)\right]=0
$$

if $N=[2 \pi / h]$.

Proof. From (11),

$$
\theta_{i}=\theta+j h+\sum_{k=0}^{i-1} h f_{2}\left(r_{k}, \theta_{k}\right)+h^{2} F_{2}\left(r_{k}, \theta_{k}, h\right) .
$$

Therefore,

$$
a\left(\theta_{i}\right)=a(\theta+j h)+a^{\prime}\left(\phi_{i}\right) \sum_{k=0}^{i-1} h f_{2}\left(r_{k}, \theta_{k}\right)+h^{2} F_{2}\left(r_{k}, \theta_{k}, h\right)
$$

for some $\phi_{j}$ between $\theta$ and $\theta+j h$. This implies that

$$
\begin{aligned}
\left|h a\left(\theta_{i}\right)-h a(\theta+j h)\right| & \leq h c \sum_{k=0}^{i-1}\left[h c(1+2 h c)^{k}(|r|+h)+c h^{2}\right] \\
& \leq h^{2} c^{2}\left[j h+\frac{(1+2 h c)^{i}}{2 h c}(|r|+h)\right] .
\end{aligned}
$$

Hence,

$$
\begin{aligned}
\sum_{i=1}^{N-1}\left|h a\left(\theta_{j}\right)-h a(\theta+j h)\right| & \leq h^{3} c^{2} \frac{N(N-1)}{2}+\frac{h c}{2}(|r|+h)\left[\frac{(1+2 h c)^{N}-1}{2 h c}\right] \\
& \leq 2 \pi^{2} c^{2} h+\frac{e^{4 \pi c}}{4}(|r|+h),
\end{aligned}
$$

and this quantity approaches zero as $|r|$ and $h$ approach zero.

Finally, observe that

$$
\lim _{h \rightarrow 0} h \sum_{i=0}^{N-1} a(\theta+j h)=\int_{0}^{2 \pi} a(\theta) d \theta .
$$

It now follows immediately from Lemmas 2 and 3 that we can find a number $\rho$ with $0<\rho<1$ such that

$$
|\lambda(\theta, r, h)| \leq \rho
$$

if $\left|\mu_{2}\right|<1$.

Proof of Theorem 1. Let $B$ be the Banach space of continuous curves $r=g(\theta)$ with $g(\theta+2 \pi)=g(\theta),\|g\|=\max \mid g(\theta)$, and $|g(\theta)| \leq \delta$, for some appropriate $\delta$. We will show that the mapping $M^{N}$ takes this space into itself, and is a contraction on this space. It will then follow immediately that the mapping $M^{N}$ has a closed invariant curve $\Gamma$ near the circle $r=0$.

Lemma 4: Let $l=4 \pi \rho c \exp (12 \pi c)$, and let $\delta$ satisfy the inequality 


$$
\frac{\rho \delta}{1-h c}+l\left(h+\delta^{2}+h^{2}\right) \leq \delta .
$$

Then, both $M^{N}$ and $M^{N-1}$ take $B$ into itself.

Proof: Suppose that $|r| \leq \delta$. Then, from (12) and (13),

$$
\begin{aligned}
\left|r_{N}\right| & \leq \rho \delta+\rho \sum_{j=0}^{N-1} \frac{c h^{2}+c h r_{i}^{2}}{(1-h c)^{j+1}} \\
& \leq \rho \delta+\frac{2 \pi \rho c h}{(1-h c)^{2 \pi / h}}+\frac{2 \pi \rho c \exp (8 \pi c)}{(1-h c)^{2 \pi / h}}(\delta+h)^{2} .
\end{aligned}
$$

Now, for $h$ small, $1-h c \geq \exp (-2 h c)$. Hence,

$$
\begin{aligned}
\left|r_{N}\right| & \leq \rho \delta+\frac{2 \pi \rho c h}{\exp (-4 \pi c)}+2 \pi \rho c \exp (12 \pi c)(\delta+h)^{2} \\
& \leq \rho \delta+l\left(h+\delta^{2}+h^{2}\right) .
\end{aligned}
$$

Thus $M^{N}$ takes $B$ into itself if

$$
\rho \delta+l\left(h+\delta^{2}+h^{2}\right) \leq \delta .
$$

However, in proving that $M$ also has a closed invariant curve, we will need that $M^{N-1}$ also takes $B$ into itself. Now it is casily verified that

$$
\left|r_{N-1}\right| \leq \frac{\rho}{1-h c} \delta+l\left(h+\delta^{2}+h^{2}\right)
$$

if $|r| \leq \delta$. Hence, if $\delta$ satisfies (15), then $B$ is invariant under both $M^{N}$ and $M^{N-1}$. Finally we observe that $\delta$ must lie between $\delta_{-}$and $\delta_{+}$, where

$$
\delta_{+}=\frac{1-\frac{\rho}{1-h c} \pm\left[\left(\frac{\rho}{1-h c}-1\right)^{2}-4 l\left(l h+l h^{2}\right)\right]^{1 / 2}}{2 l} .
$$

Hence we can find a suitable $\delta$ if we choose $h$ so small so that

$$
4 l\left(l h+l h^{2}\right) \leq\left(\frac{\rho}{1-h c}-1\right)^{2} .
$$

Lemma 5: The mapping $M^{N}$ is a contraction on the space $B$, for $h$ and $\delta$ suitably small.

Proof: Let $r=g(\theta)$ be a curve in $B$. The image of this curve is the curve $r=g_{1}(\theta)$, where (see Eq. (12))

$$
\lambda(\theta, g(\theta), h) g(\theta)+F(g(\theta), \theta, h)=g_{1}(\theta+G(g(\theta), \theta, h)) .
$$

Equivalently,

$$
g_{1}(\theta)=\lambda\left(\theta_{-N}, g\left(\theta_{-N}\right), h\right) g\left(\theta_{-N}\right)+F\left(g\left(\theta_{-N}\right), \theta_{-N}, h\right) .
$$

If $r=\hat{g}(\theta)$ is a second curve in $B$, then its image under $M^{N}$ is $r=\hat{g}_{1}(\theta)$ where

$$
\hat{g}_{1}(\theta)=\lambda\left(\hat{\theta}_{-N}, \hat{g}\left(\hat{\theta}_{-N}\right), h\right) \hat{g}\left(\hat{\theta}_{-N}\right)+F\left(\hat{g}\left(\hat{\theta}_{-N}\right), \hat{\theta}_{-N}, h\right) .
$$

(Notice that $\hat{\theta}_{-N}$ depends on $\hat{g}(\theta)$.) We want to show that

$$
\left\|g_{1}(\theta)-\hat{g}_{1}(\theta)\right\| \leq \sigma\|g(\theta)-\hat{g}(\theta)\|
$$


for some constant $\sigma$, with $0 \leq \sigma<1$. To this end we first prove that $\partial r_{k} / \partial r$ and $\partial \theta_{k} / \partial r$ are bounded, for $1 \leq k \leq N-1$.

Lemma 6: The partial derivatives $\partial r_{k} / \partial r$ and $\partial \theta_{k} / \partial r, k=1, \cdots, N-1$, are uniformly bounded for $r$ and $h$ small.

Proof: Observe that

$$
\left(\begin{array}{l}
\partial r_{k} / \partial r \\
\partial \theta_{k} / \theta r
\end{array}\right)=\left(\begin{array}{ll}
\partial r_{k} / \partial r_{k-1} & \partial r_{k} / \partial \theta_{k-1} \\
\partial \theta_{k} / \partial r_{k-1} & \partial \theta_{k} / \partial \theta_{k-1}
\end{array}\right)\left(\begin{array}{l}
\partial r_{k-1} / \partial r \\
\partial \theta_{k-1} / \partial r
\end{array}\right)
$$

so that

$$
\left(\begin{array}{l}
\partial r_{k} / \partial r \\
\partial \theta_{k} / \partial r
\end{array}\right)=\prod_{i=2}^{k}\left(\begin{array}{ll}
\partial r_{i} / \partial r_{i-1} & \partial r_{i} / \partial \theta_{i-1} \\
\partial \theta_{i} / \partial \theta_{i-1} & \partial \theta_{i} / \partial \theta_{i-1}
\end{array}\right)\left(\begin{array}{l}
\partial r_{1} / \partial r \\
\partial \theta_{1} / \partial r
\end{array}\right)
$$

Choose $\alpha$ so large that

$$
\left\{\begin{array}{llll}
\left|\partial f_{1} / \partial r\right| \leq \alpha|r|, & \left|\partial F_{1} / \partial r\right| \leq \alpha, & \left|\partial f_{2} / \partial r\right| \leq \alpha, & \left|\partial G_{1} / \partial r\right| \leq \alpha \\
\left|\partial f_{1} / \partial \theta\right| \leq \alpha|r|^{2}, & \left|\partial F_{1} / \partial \theta\right| \leq \alpha, & \left|\partial f_{2} / \partial \theta\right| \leq \alpha|r|, & \left|\partial G_{1} / \partial \theta\right| \leq \alpha .
\end{array}\right.
$$

Then,

$$
\begin{aligned}
& \left|\partial r_{i} / \partial r_{i-1}\right| \leq 1+h c+h \alpha \exp (4 \pi c)[|r|+h]+\alpha h^{2}, \\
& \left|\partial r_{i} / \partial \theta_{i-1}\right| \leq h c \exp (4 \pi c)[|r|+h]+h \alpha \exp (8 \pi c)[|r|+h]^{2}+\alpha h^{2}, \\
& \left|\partial \theta_{i} / \partial r_{i-1}\right| \leq h \alpha+\alpha h^{2}, \\
& \left|\partial \theta_{i} / \partial \theta_{i-1}\right| \leq 1+h \alpha \exp (4 \pi c)[|r|+h]+\alpha h^{2} .
\end{aligned}
$$

Consequently,

$$
\left(\begin{array}{c}
\left|\partial r_{k} / \partial r\right| \\
\left|\partial \theta_{k} / \partial r\right|
\end{array}\right) \leq\left(\begin{array}{cc}
1+\beta h & \beta h \\
\beta h & 1+\beta h
\end{array}\right)^{k-1}\left(\begin{array}{c}
1+\beta h \\
\beta h
\end{array}\right)
$$

for some suitable constant $\beta$. Using the fact that $\left\|A^{i}\right\| \leq\|A\|^{i}$ for any matrix $A$, and

$$
\left\|\left(\begin{array}{cc}
1+\beta h & \beta h \\
\beta h & 1+\beta h
\end{array}\right)\right\|=1+2 \beta h .
$$

we see that

$$
\begin{aligned}
\max \left\{\left|\partial r_{k} / \partial r\right|,\left|\partial \theta_{k} / \partial r\right|\right\} & \leq(1+2 \beta h)^{k-1}(1+\beta h) \\
& \leq(1+2 \beta h)^{k} \leq \exp (2 \beta h k) \leq \exp (4 \pi \beta)
\end{aligned}
$$

and this proves Lemma 6 .

Lemma 6, together with the estimate $|\lambda(\theta, r, h)| \leq \rho<1$ and the observation that $\left|\theta_{-N}-\hat{\theta}_{-N}\right|$ is very small compared to $|g(\theta)-\hat{g}(\theta)|$, gives us the estimate (16), and this completes the proof of Lemma 5 .

It follows immediately from Lemma 5 that $M^{N}$ has a closed invariant curve $\Gamma$ near the circle $r=0$ if $\mu_{2}<1$. This in turn implies that the curves $M \Gamma, M^{2} \Gamma, \cdots, M^{N-1} \Gamma$ are also invariant under $M^{N}$. As remarked previously, the curve $M^{N-1} \Gamma$ lies in $B$. However, since $M^{N}$ is a contraction on $B$, the curves $\Gamma$ and $M^{N-1} \Gamma$ must be the same, and this can only be the case if $M \Gamma=\Gamma$. Thus $\Gamma$ is also invariant under $M$. Finally, if $\mu_{2}>1$, 
then we work with $M^{-1}$ instead of $M$, and the proof goes through exactly as before. This completes the proof of Theorem 1 in the special case $n=2$.

Remarks: 1. Our proof of Theorem 1 generalizes quite easily to the case $n>2$. Let $x=\phi(t)$ be a periodic solution of $\dot{x}=f(x)$ with characteristic multipliers $\mu_{1}, \cdots, \mu_{n-1}$, $\mu_{n}=1$. Then, we can find new coordinates $r, \theta$ such that

$$
\dot{r}=A(\theta) r+f_{1}(r, \theta), \quad \dot{\theta}=1+f_{2}(r, \theta)
$$

and, moreover, the periodic system $\dot{r}=A(t) r$ has characteristic multipliers $\mu_{1}, \cdots, \mu_{r-1}$. The only change in the proof of Theorem 1 is in estimating the quantity $\lambda=\prod_{i=1}^{N-1}[1+$ $\left.h A\left(\theta_{i}\right)\right]$. Here, we must appeal to our knowledge that the solutions of the finite difference scheme converge to the solutions of the differential equation as $h \rightarrow 0$.

2. Using our knowledge that the solutions of the finite difference scheme converge to the solutions of the differential equations, we can greatly sharpen the estimates in this proof. Thus, the mapping $M$ has a closed invariant curve for much larger values of $h$ than our proof implies.

Application to the Van der Pol equation. It is well known (Hale [4]) that the periodic solution of Van der Pol's equation (5) is orbitally asymptotically stable. This follows from the Poincaré-Bendixson Theorem. To apply Theorem 1 to this equation, we need the additional fact that $\mu_{2}<1$. We prove this as follows: Set

$$
y=\left(\begin{array}{l}
x \\
\dot{x}
\end{array}\right) .
$$

Then, the variational equation of (5) about the periodic solution

$$
y=\left(\begin{array}{r}
2 \cos t \\
-2 \sin t
\end{array}\right)+O(\epsilon)
$$

is

$$
\dot{\zeta}=\left(\begin{array}{cc}
0 & 1 \\
-1-2 \epsilon \dot{x} & -\epsilon\left(x^{2}-1\right)
\end{array}\right) \zeta .
$$

To first order in $\epsilon$, the determinant of the monodromy matrix $\Omega$ of (17) is

$$
\begin{aligned}
\operatorname{det} \Omega & =\exp \left(-\epsilon \int_{0}^{2 \pi}\left[4\left(\frac{1}{2}+\frac{\cos 2}{2} \frac{t}{2}\right)-1\right] d t+O\left(\epsilon^{2}\right)\right) \\
& =\exp \left(-2 \pi \epsilon+O\left(\epsilon^{2}\right) .\right.
\end{aligned}
$$

Hence, det $\Omega<1$ for $\epsilon>0$ sufficiently small, and this implies that $\mu_{2}<1$. Consequently, every finite difference approximation of the Van der Pol equation which is at least as accurate as Euler's method has a closed invariant curve near the circle of radius 2 for $\epsilon>0$ and $h$ sufficiently small.

The inverse problem. In this section we show that the existence of a closed invariant curve $\Gamma$ of a finite difference approximation implies the existence of a periodic solution of the differential equation. Indeed, we reverse our steps. Assume that the difference 
approximation

$$
x_{1}=x+h f(x)+h^{2} F(x, h)
$$

of the differential equation $\dot{x}=f(x)$ has a closed invariant curve $\Gamma$ for some value $h$, and that we can find new coordinates $r, \theta$ such that $r=0$ is $\Gamma$ and

$$
\begin{aligned}
& r_{1}=r+h A(\theta) r+h f_{1}(r, \theta, h) \\
& \theta_{1}=\theta+h g(\theta)+h g_{1}(r, \theta, h)
\end{aligned}
$$

with $g(\theta)>0$ and $f_{1}$ and $g_{1}$ small compared to $h$. If we write the differential equation in terms of $r$ and $\theta$, we find that

$$
\dot{r}=A(\theta) r+h F_{1}(r, \theta, h), \quad \dot{\theta}=g(\theta)+h G_{1}(r, \theta, h) .
$$

Define a new time scale $\tau$ by the relation $d t / d \tau=1 / g(\theta)$. Then,

$$
\begin{aligned}
& d r / d \tau=(A(\theta) / g(\theta)) r+h F_{1} / g(\theta) \\
& d \theta / d \tau=1+h G_{1} / g(\theta)
\end{aligned}
$$

If all the characteristic multipliers of the system

$$
\dot{\zeta}=(A(t) / g(t)) \zeta
$$

have modulus $\neq 1$, then the system (18) has a periodic solution if $h$ is sufficiently small. This follows immediately from Poincaré's continuation theroem.

\section{REFERENCES}

[1] M. Braun, Differential equations and their applications, A.M.S. series \# 15, Springer-Verlag, 1975

[2] A. Halanay, Invariant manifoids for discrete systems, in Proceedings of the second Czechoslovakian conference on differential equations and their applications (Equadiff II), Bratislava, Czechoslovakia, 1966

[3] A. Halanay, Periodic and almost periodic solutions of systems of finite difference equations, Arch. Rat. Mech. Anal. 12, pp. 134-149 (1963)

[4] J. Hale, Differential equations, Wiley-Interscience, 1969

[5] J. Hurt, Almost periodic solutions of difference equations, Ph.D. dissertation, Brown University, 1968 